\title{
ESTIMACIÓN ESTADÍSTICA DE LA RELACIÓN ENTRE GASTOS DE PUBLICIDAD Y SU IMPACTO EN EL DESEMPEÑO FINANCIERO
}

\author{
STATISTICAL ESTIMATION OF THE RELATIONSHIP BETWEEN \\ ADVERTISING EXPENSES AND THEIR IMPACT ON FINANCIAL \\ PERFORMANCE
}

\author{
César Enrique Freire Quintero, ${ }^{1}$ Victor Hugo Calle Armijos, ${ }^{1}$ José Patricio Barre Delgado, ${ }^{1}$ Cris- \\ thian Armando Holguín León, ${ }^{1}$ Cristhian Daniel Pacheco Parrales ${ }^{1}$
}

1 Facultad de Economía Agrícola, Universidad Agraria del Ecuador, Guayaquil - Ecuador.

E-mail: cfreire@uagraria.edu.ec; vcalle@uagraria.edu.ec; barrleon171996@hotmail.com; crisholguin97@hotmail.com; pkriss-pacheco@hotmail.com

\section{RESUMEN}

El presente trabajo analiza el impacto de los gastos de publicidad en el desempeño financiero, considerando que la publicidad es un activo intangible asociado a las ventas y que informa al consumidor sobre la existencia de productos y sus precios. Para analizar el impacto que ejerce el gasto de publicidad sobre el desempeño financiero de 296 empresas dentro del territorio ecuatoriano estudiadas se utilizó el método de regresión lineal múltiple; además, para suavizar la dispersión de los datos se utilizó LN (Logaritmo Natural) en la variable dependiente ROA (Rentabilidad de los Activos) y en las variables independientes de Publicidad, Pasivo, e Ingresos totales. Dentro de los resultados se determinó que el sesgo estandarizado y la Curtosis estandarizada, se encuentran dentro del rango esperado para datos provenientes de una distribución normal. En el mismo sentido la correlación de Pearson entre cada variable, determinó la correlación en cuatro pares de variables. Además, los coeficientes de Tolerancia de las variables están próximos a la unidad y el FIV = Factor de Inflación de la Varianza de las variables no es mayor de 10. Por último, se concluye que, con un nivel de confianza del $95 \%$, no existe relación estadísticamente significativa entre la variable publicidad y desempeño financiero.

PALABRAS CLAVE: desempeño financiero, publicidad, regresión, statgraphics, spss
The following research article analyzes the impact of advertising expenses on financial performance, considering that advertising is an intangible asset associated with sales and informs the consumer about the existence of products and their prices. To analyze the impact of advertising spending on the financial performance of 296 companies within the Ecuadorian territory studied, the multiple linear regression method was used; In addition, to smooth the dispersion of the data, LN (natural logarithm) was used in the dependent variable ROA (Return on Assets) and in the independent variables of Advertising, Passive, and Total Income. Within the results it was determined that the standardized bias and standardized Kurtosis are within the expected range for data from a normal distribution. In the same sense, the Pearson correlation between each variable determined the correlation in four pairs of variables. In addition, the Tolerance coefficients of the variables are close to unity and the IVF = Variation Inflation Factor of the variables is not greater than 10. Finally, it is concluded that, with a confidence level of $95 \%$, there is no statistically significant relationship between the advertising variable and financial performance.

KEYWORDS: financial performance, advertising, regression, stat graphics, spss 


\section{Introducción}

La publicidad es una rama de las comunicaciones del proceso de mercadotecnia (Zurita, 2015) y es a través la comunicación y la propaganda, que un proveedor informa y motiva a la sociedad, o a un segmento de ésta, a adquirir o contratar el bien o servicio que ofrece (Ricalde, 2013).

De acuerdo a Zhang (2016), la publicidad se puede ejecutar mediante medios masivos como volantes, revistas, prensa, internet y radio para difundir información específica sobre bienes y servicios, por lo mismo, el importe de los gastos satisfechos por estos conceptos determinan la denominación de estas cuentas (Velez, 2014).

Sobre la base de lo indicado, el crecimiento de una empresa está acompañado por el incremento en el gasto de insumos publicitarios los que aumentan posteriormente su producción ( Beltrán, 2016).

Por otra parte, el desempeño financiero es uno de los indicadores que se utilizan para medir el éxito en términos de su rentabilidad, la que, de acuerdo con Castillo y García (2013) está en función factores como su infra estructura, tamaño y estructura financiera (p. 152).

En esta misma línea de análisis, según Cárdenas y Velasco (2013), la sostenibilidad financiera, está dada por la capacidad para operar y crecer, alcanzar el equilibrio de sus activos y pasivos y garantizar su rentabilidad y liquidez" (p. 22).

Se trata entonces de mantener controlado el desempeño financiero mediante el análisis financiero integral de los estados, la competitividad y la capacidad que tienen la empresa de crear valor y atraer nueva inversión (Castaño \& Arias, 2014).

Por una parte, el capital físico que determina el ROA de las empresas (Joshi, Cahill, Sidhu, \& Kansal, 2013, p. 279) y, por otra, el nivel de deuda sin embargo, las empresas rentables están menos preocupadas por su nivel de deuda (Zeitum \& Salman, 2015) que por el incremento de sus inversiones.

En síntesis, el desempeño financiero es usado como una herramienta que nos permite medir las fortalezas y debilidades de las empresas sobre la base del análisis de sus activos y pasivos, sus ventas y sus cuentas por cobrar (Vanessa Pineda Bulnes, 2015), muchas de ellas provenientes de sus ventas, en consecuencia, asociadas a las inversiones en publicidad (Vasquez, 2016).

De acuerdo a los autores (Ross, Westerfield, \& Jaffe, 2012) existen varias alternativas para determinar el desempeño financiero de las empresas, prueba de ello son dos indicadores de gran utilidad, estos son el Retorno sobre los activos (ROA) y el Retorno sobre el capital (ROE). En este caso en particular la evaluación del desempeño financiero será evaluada utilizando el ROA de las empresas.

Se busca predecir el comportamiento de una variable a partir de otra mediante el análisis de datos históricos, considerando una relación lineal ajustada de datos para estimar el mejor ajuste entre estas variables (Cardona, González, Rivera, \& Cárdenas, 2013).

Se ha evidenciado la necesidad de explicar el desempeño financiero de las empresas a través de varias variables, entre algunas de las más relevantes están las que recomendaron (Langebaek \& Ortiz, 2007), las cuales son: (a) Publicidad, (b) Nivel de endeudamiento e (c) Indice de Gobierno Corporativo (IGC).

Como mencionan (Langebaek \& Ortiz, 2007), se pueden diferenciar dos tipos de indicadores para medir el efecto de la publicidad: indicadores de insumo (inversión en publicidad) e indicadores de impacto. Al igual que estos autores, por disponibilidad de información recurrimos a un indicador de insumo: la inversión en publicidad.

Sobre la base de lo analizado, el presente trabajo analiza entonces, la relación que se produce entre los gastos de publicidad respecto del resultado financiero de las empresas (Cambridge University Press, 2009), a fin de conocer la relación o grado de asociación que existe entre dos o más variables, la que puede ser positiva o negativa (Freire, Morán, Macas, \& Paucar, 2015).

\section{Métodología}

Para esta investigación se usó información proveniente de la Superintendencia de Compañías agrupada en una base de datos con información sobre el nivel de pasivos, ingresos totales y publicidad de 296 empresas dentro del territorio ecuatoriano, se estableció una base de datos, con la cual se trabajó el modelo de regresión lineal múltiple para determinar la afectación de estas variables sobre el ROA. De modo que para el análisis mencionado se usarán los programas estadísticos tales como STATGRAPHICS y SPSS.

Respecto del análisis de regresión, se tuvo en consideración los argumentos de Locia, Mederos, Morales y Rodríguez (2014), quienes mencionaron que entre las características de la ecuación lineal se encuentran la pendiente de la recta y la localización de la recta en algún punto. La regresión lineal simple también se manejó bajo supuestos para determinarla. Además, Caligaris, Rodríguez y Lauguero (2014) mencionaron la necesidad del uso de los supuestos de (i) Normalidad, (ii) homocedasticidad, (iii) independencia del error y (iv) linealidad; finalmente, que el modelo de regresión lineal es el apropiado para realizar pronósticos cuando la tendencia es lineal (Guerrero, 2016). 
La regresión tiene por objeto poner de manifiesto la estructura de dependencia que mejor explique el comportamiento de una variable Y (variable dependiente o explicada) a través de un conjunto de variables (variables independientes o explicativas), con las que se relaciona (García Ramos, Ruiz Garzón, \& Ramos, 2016). El análisis de regresión que permite establecer la relación funcional o ecuación matemática que relaciona las variables, así como la fuerza de esa relación (Cardona, González, Rivera, \& Cárdenas, 2013).

Complementariamente, con relación a la regresión lineal múltiple, lo más frecuente es que tanto la variable dependiente como las independientes sean variables continuas medidas en escala de intervalo o razón (Samaniego \& Buenahora, 2016). Por otra parte, en el modelo de RLM, que puede ser en base a una variable endógena o una transformación de las variables endógenas, las que se transforman en una función lineal de $\mathrm{k}$ represores correspondientes a las variables explicativas y una perturbación aleatoria o error. (Uriel , 2013).

Se utiliza k para representar el número de variables independientes. Por lo tanto, $\mathrm{k}$ puede ser cualquier número entero positivo (Lind, Marchal, \& Wathen, 2012). Este modelo se presenta cuando dos o más variables independientes influyen sobre una variable dependiente (Rodriguez y Jimenez, 2016, p. 370). Al igual que en regresión lineal simple, los coeficientes b van a indicar el incremento en el valor por el incremento unitario de la correspondiente variable explicativa. Por lo tanto, estos coeficientes van a tener las correspondientes unidades de medida (Rojo, 2007).

$$
\hat{\mathrm{y}}=\mathrm{a}+\mathrm{b}_{1}{ }^{*} \mathrm{x}_{1}+\mathrm{b}_{2}{ }^{*} \mathrm{x}_{2}+\mathrm{b}_{3}{ }^{*} \mathrm{x}_{3}+\ldots+\mathrm{b}_{\mathrm{k}}{ }^{*} \mathrm{x}_{\mathrm{k}}+\varepsilon
$$

De acuerdo a Sáez (2012), (Y) representa, a la variable dependiente, que deseamos estudiar en relación con otras. (X) representa a la variable independiente que puede afectar en alguna medida a la variable dependiente. Ro- mero y Zunica (2013) comparten que, (a) es la intersección de (Y) y b_1 es el cambio neto en $\mathrm{Y}$ por cada cambio unitario en $\mathrm{X} 1$, manteniendo $\mathrm{X} 2$ constante, se denomina coeficiente de regresión parcial, coeficiente de regresión neta o bien coeficiente de regresión y $\varepsilon$ es el error estándar.

Se trabajó el Error estándar, considerando una medida de dispersión de la estimación acorde con el plano de regresión más pequeño (Menchaca, 2012).Se considera la desviación estándar de todas las muestras posibles de la población, medida que se la conoce también como una desviación estándar que ha sido calculada de una muestra de datos que está siendo estudiada en ese mismo momento (Galarza, 2015).

$$
\mathrm{S}_{\mathrm{xy}}=\frac{\sum(\mathrm{Y}-\hat{\mathrm{y}})}{\mathrm{n}-\mathrm{m} .1}
$$

Y: Valores observados en la muestra;

$\hat{y}$ : Valores estimados a partir a partir de la ecuación de regresión;

$\mathrm{n}$ : Número de datos y;

$\mathrm{m}$ : Número de variables independientes (Cardona, González, Rivera, \& Cárdenas, 2013).

El coeficiente de determinación múltiple

Mide la tasa porcentual de los cambios de $Y$ que pueden ser explicados por $x^{\wedge} 1, x^{\wedge} 2 \mathrm{y} \mathrm{x}^{\wedge} 3$ simultáneamente (Joekes, Righetti, \& Yacci, 2016).

$$
\mathrm{r}^{2}=\frac{\text { SCregresion }}{\text { SCTotal }}
$$

\section{Resultados}

Por medio de la recopilación de información sobre el nivel de pasivos, ingresos totales y publicidad de 296 empresas dentro del territorio ecuatoriano (Tabla 1), se generó una base de datos con la cual se trabajó el modelo de regresión lineal múltiple para determinar la afectación de estas variables sobre el ROA.

\begin{tabular}{|c|c|c|c|c|}
\hline & LN ingresos & LN publicidad & LN pasivo & LNROA \\
\hline Recuento & 296 & 296 & 296 & 296 \\
\hline Promedio & 15,337 & 6,06655 & 13,5516 & $-2,94628$ \\
\hline Mediana & 15,3346 & 6,09387 & 13,5521 & $-2,94358$ \\
\hline Varianza & 1,83502 & 3,46838 & 1,82964 & 1,14638 \\
\hline \multicolumn{5}{|l|}{ Desviación } \\
\hline $\begin{array}{l}\text { Estándar } \\
\text { Coeficiente }\end{array}$ & 1,35463 & \multicolumn{2}{|c|}{ Coeficiente de } & 1,07069 \\
\hline Variación & $8,83 \%$ & $30,70 \%$ & $9,98 \%$ & $-36,34 \%$ \\
\hline Error Estándar & $7,87 \%$ & $10,82 \%$ & $7,86 \%$ & 0,0622328 \\
\hline Mínimo & 11,8326 & 1,33479 & 10,0139 & $-5,72194$ \\
\hline Máximo & 18,864 & 10,7421 & 16,8684 & $-0,156108$ \\
\hline Rango & 7,03137 & 9,40733 & 6,85458 & 5,56583 \\
\hline Sesgo & & & & \\
\hline $\begin{array}{l}\text { Estandarizado } \\
\text { Curtosis }\end{array}$ & $-0,0662743$ & $-0,335272$ & $-0,230601$ & $-0,0649376$ \\
\hline Estandarizada & $-1,0476$ & $-1,16324$ & $-1,23189$ & $-0,893199$ \\
\hline
\end{tabular}

Tabla1: Resumen estadístico de las variables estudiadas, están especificadas medidas de tendencia central, variabilidad y de forma. 
Sobresalen el sesgo estandarizado y la Curtosis estandarizada, herramientas necesarias, utilizadas para precisar si la muestra proviene de una distribución normal; es decir, si ayuda a identificar si los datos del modelo se encuentran dentro de los rangos normales de una distribución normal $(-2 \mathrm{a}+2)$ y si las variables se encuentran fuera de este rango indican desviaciones significativas, lo cual significa un problema en el modelo e invalidaría muchos de los procedimientos estadísticos a utilizar.

El sesgo estandarizado y la curtosis estandarizada en cada una de las variables indican que los datos se encuentran asimétricos respecto a la media, por lo que mantiene una gran dispersión.

Para suavizar la dispersión de los datos se utilizó LN en las variables de Publicidad, Pasivo, ROA e Ingresos totales (Tabla 2).

Tabla 2 : Resumenestadístico pararesiduos

\begin{tabular}{ll}
\hline Recuento & 296 \\
\hline Promedio & $5,43581 \mathrm{E}-8$ \\
Desviación Estándar & 0,922526 \\
Coeficiente de Variación & $1,69713 \mathrm{E} 9 \%$ \\
Mínimo & $-2,34109$ \\
Máximo & 2,35913 \\
Rango & 4,70022 \\
Sesgo Estandarizado & $-0,336645$ \\
Curtosis Estandarizada & $-1,04977$ \\
\hline
\end{tabular}

En este caso se puede observar valores de sesgo estandarizado y de Curtosis estandariza$\mathrm{da}$, con valores fuera de rango $(-2 \mathrm{a}+2)$ indican desviaciones significativas a la normalidad, lo que tendería a invalidar cualquier prueba estadística con referencia a la desviación estándar. Sin embargo, el valor del sesgo estandarizado se encuentra dentro del rango esperado para datos provenientes de una distribución normal.

El valor de Curtosis estandarizada por su parte, se encuentra dentro del rango esperado para datos provenientes de una distribución normal.

Tabla 3: Correlación de Pearson en las variables estudiadas

\begin{tabular}{lllll}
\hline & LN INGRESOS & LN PUBLICIDAD & LN PASIVO & LN ROA \\
\hline LN INGRESOS & & 0,3264 & 0,8013 & 0,0809 \\
& & 296 & 296 & 296 \\
LN PUBLICIDAD & 0,3264 & 0 & 0 & 0,1651 \\
& 296 & & 0,2735 & $-0,0403$ \\
& 0 & & 296 & 296 \\
LNPASIVO & 0,8013 & 0,2735 & & 0,4898 \\
& 296 & 296 & & $-0,2328$ \\
LNROA & 0 & 0 & & 296 \\
& 0,0809 & $-0,0403$ & $-0,2328$ & 0,001 \\
& 296 & 296 & 296 & \\
\hline
\end{tabular}

La correlación de Pearson entre cada variable, determina la Correlación, Tamaño de Muestra y Valor-P. El rango de estos coeficientes de correlación va de -1 a +1 , y miden la fuerza de la relación lineal entre las variables en estudio. Para este caso el número referencial de cada bloque es el valor-P, que prueba la significancia estadística de las correlaciones estimadas. Seguido de los valores-P abajo de o,05 indican correlaciones significativamente diferentes de cero, con un nivel de confianza del $95,0 \%$.

Finalmente los siguientes pares de variables tienen valores-P por debajo de o,05: (1) LN ingreso total y LN pasivo, (2) LN ingreso total y LN publicidad, (3) LN pasivo y LN publicidad y (4) LN pasivo y LN ROA.

Tabla4: Regresión Múltiple LN ROA

\begin{tabular}{lllll}
\hline & & Error & Estadístico & \\
\hline Parámetro & Estimación & Estándar & T & Valor -P \\
CONSTANTE & $-3,11637$ & 0,620097 & $-5,02561$ & 0,0000 \\
LN PUBLICIDAD & $-0,0365334$ & 0,0306752 & $-1,19098$ & 0,2346 \\
LN INGRESOS & 0,605639 & 0,067805 & 8,93206 & 0,0000 \\
LNPASIVO & $-0,656525$ & 0,0667298 & $-9,83855$ & 0,0000 \\
\hline
\end{tabular}


Se realizó la regresión múltiple en función de la variable dependiente " $y$ " LN ROA; y las variables independientes " $x$ " LN Ingreso Total, LN Pasivo, LN Publicidad. Los resultados obtenidos después de ajustar un modelo de regresión lineal múltiple para describir la relación entre LN ROA y 3 variables independientes. La ecuación final del modelo ajustado es:

LN ROA $=-3,11637-0,0365334^{*} \mathrm{LN}$ publicidad $+0,605639^{*} \mathrm{LN}$ ingresos - o, 656525*N

Sin embargo, se pudo detectar según la información obtenida en la tabla 4 , que no todos los "Valor-P" de las variables son mayor que el $5 \%$ o o,05. Se determina que sí existe una relación entre las variables de Nivel de LN ingresos y LN Pasivo con la variable independiente LN $\mathrm{ROA}$, en base a esto se puede determinar que sí existe una relación estadísticamente significativa entre esas dos variables con un nivel de confianza del 95,0\%.
Por otra parte LN Publicidad tiene un "Valor-P" de $5 \%$ o o,05, lo que determina que no existe una relación entre esta variable y la variable dependiente LN ROA, en base a esto se puede determinar que no existe una relación estadísticamente significativa entre las variables con un nivel de confianza del 95,0\%.

Los coeficientes de Tolerancia deben estar próximos a la unidad ya que $\mathrm{Si}<0,1$, es problemático, debido a que al darse ese comportamiento no cumple con la colinealidad. En este análisis todas las variables cumplen este supuesto. El FIV = Factor de Inflación de la Varianza, no debe ser mayor de 10, ya que resulta problemático, debido a que este representa la proporción de la variabilidad de la variable que es explicada por el resto de las variables predictoras del modelo. Como es lógico, cuanto mayor sea el factor de inflación de la varianza, mayor será la probabilidad de que exista colinealidad. Las variables estudiadas cumplen la regla de no ser mayores a 10.

Tabla5: Coeficientes de regresión

\begin{tabular}{|c|c|c|c|c|c|c|c|c|}
\hline \multicolumn{9}{|c|}{ Coeficientes } \\
\hline \multirow{2}{*}{\multicolumn{2}{|c|}{ Modelo }} & \multicolumn{2}{|c|}{$\begin{array}{l}\text { Coeficientes no } \\
\text { estandarizados }\end{array}$} & \multirow{2}{*}{$\begin{array}{l}\text { Coeficientes } \\
\text { tipificados } \\
\text { Beta }\end{array}$} & \multirow[t]{2}{*}{$\mathrm{t}$} & \multirow[t]{2}{*}{ Sig. } & \multicolumn{2}{|l|}{$\begin{array}{l}\text { Estadísticos } \\
\text { colinealidad }\end{array}$} \\
\hline & & $\mathrm{B}$ & Error típ. & & & & Tolerancia & FIV \\
\hline & LNINGRESO & 0,603 & 0,073 & 0,702 & 8,246 & o & 0,351 & 2,853 \\
\hline & LNPUBLICIDAD & $-0,041$ & 0,027 & $-0,082$ & $-1,537$ & 0,125 & 0,883 & 1,132 \\
\hline
\end{tabular}

Discusión

De acuerdo a los resultados de Zeitum y Salman (2015), para analizar la relación gasto en publicidad y sus efectos en el desempeño financiero de las empresas se debe aplicar un modelo de regresión logística en el cual se considere el análisis de más variables tales como: $\mathrm{ROA}=\beta \_0+\beta \_1$ Ratio Liquidez $+\beta \_2$ Nivel de endeudamiento $+\beta_{-3}$ Crecimiento Ventas. En donde la variable dependiente es la rentabilidad económica.

$\mathrm{Si}$ el gasto extra en publicidad es menor o igual que los beneficios incrementales $(A+B$ $+C)$, entonces se puede decir que la publicidad se paga. Si los beneficios aumentan más que los gastos en publicidad entonces se deben seguir aumentando la inversión publicitaria.

La táctica impulsora incrementará las ventas de una empresa, en un porcentaje mensual en las negociaciones de los productos de la empresa, estableciendo estrategias promocionales que puedan implementarse en el corto plazo, fortaleciendo la imagen, el nombre o marca de la empresa, utilizando los medios publicitarios viales. Realizando planes de acción propuesto para la estrategia de publici- dad y el lugar de exposición de los productos. Es muy primordial monitorear la efíciencia de la estrategia para el control sobre el comportamiento de las ventas. (Chavarria, 2011)

Los sectores que invierten en publicidad (para diferenciar sus productos) tienden a tener mayores márgenes de ganancia, pero esto depende del tipo de productos que se producen, los sectores Low-Tech tienden a tener un impacto positivo del gasto en publicidad sobre la rentabilidad, mientras que para los High-Tech el gasto en tecnología implica mayores márgenes de ganancia. (Fujii, 2004)

En resumen, de acuerdo a las estimaciones realizadas en este apartado, los sectores del tipo High-Tech tienden a tener mayores ganancias para ambas muestras. En el caso de la Encuesta Industrial Anual (EIA), los sectores del tipo Low-Tech que aparentemente tienen mayor rentabilidad son aquellos que tienen una mayor concentración de mercado, están enfocados al mercado doméstico, invierten en publicidad y reciben mayores cantidades de inversión extranjera. Mientras tanto, un sector High-Tech que tiende a tener mayores ganancias invierte en publicidad y tecnología, lo cual, puede suponerse, eventualmente le permite mantenerse en el grupo High-Tech, ade- 
más de que puede ser competitivo o no. (Fujii, 2004).

La empresa analiza las variables internas (productos, precio, plaza, precio y comunicación), que son esenciales para que toda operación de negocios de cualquier bien o servicio sea eficiente y efectiva, mientras los fabricantes de productos y comercializadores finales invierten en fortunas en la calidad del producto entre una de las interrogantes planteadas se tiene: ¿por qué a las empresas industriales se les complica dar valor al posicionamiento y a la marca?, esto se debería a la falta de información que hay con respecto a la producción y ventas en una empresa industrial, y nunca es tarde para empezar a usar herramientas de otras disciplinas concluye el autor que la estrategia en mercadotecnia implica un plan para concretar.

En la actualidad las empresas buscan optimizar la rentabilidad en el mercado para alcanzar el posicionamiento y rompiendo las barreras industriales, manejar la publicidad y el éxito comercial. (Castro, 2010)

Como resultado de la investigación la variable independiente de "Publicidad" no tiene un impacto significativo dentro del desempeño financiero de las empresas estudiadas. El hecho de no haber utilizado las variables independientes indicadas por Zeitum y Salman (2015), posiblemente incidieron en el resultado final de esta investigación, la limitación de esta investigación se generó debido a que la información en la base de la Superintendencia de Compañías es incompleta por lo que se optó por escoger como variables independientes el ingreso total y el Pasivo de las empresas, en lugar de el ratio de liquidez y el nivel de endeudamiento.Además, en esta investigación no se consideró una diferenciación del tipo de empresas, sean estas de alta o baja tecnología, lo cual considerando lo indicado por Fujii (2004) permitiría obtener resultados más precisos sobre la incidencia del gasto de publicidad.

\section{Conclusiones}

De acuerdo a los resultados obtenidos se puede concluir que sí existe una relación entre las variables de Nivel de LN ingresos y LN Pasivo con la variable independiente LN ROA, en base a esto se puede determinar que sí existe una relación estadísticamente significativa entre esas dos variables con un nivel de confianza del $95,0 \%$.

Finalmente no existe relación estadísticamente significativa entre la variable publicidad y desempeño financiero al $95 \%$ de confianza; es decir la variable independiente de "Publicidad" no tiene un impacto significativo dentro del desempeño financiero de las empresas estudiadas.

\section{Referencias}

Beltrán, J. (2016). Análisis de la publicidad como industria creativa y su aporte a la economía cultural en Bogotá D.C. Bogotá: Universidad de la Salle.

Caligaris, M., Rodríguez, G., \& Lauguero, L. (2014). La formación de conceptos en la resolución numérica de ecuaciones no lineales. Argentina: Documentos en Educación Matemática.

Cambridge University Press. (2009). Fuentes de Poder de Mercado, Publicidad y Estrategias de Marketing . Cambridge: Cambridge University Press.

Cárdenas, E., Rivera, M., González, J., \& Cardona, D. (2013). Aplicación de la regresión lineal en un problema de pobreza. Revista Interacción, 73-84.

Cárdenas, M., \& Velasco, B. (2013). Incidencia de la morosidad de las cuentas por cobrar en la rentabilidad y la liquidez: estudio de caso de una Empresa Social del Estado prestadora de servicios de salud. Facultad Nacional de Salud Pública. Facultad Nacional de Salud Pública, 16-25.

Cardona, D., González, J., Rivera, M., \& Cárdenas, E. (2013). Módulo de Regresión Lineal. . Documentos de Investigación, 6o.

Castaño, C., \& Arias, J. (2014). Análisis financiero integral de empresas colombianas 2009-2012 desde la perspectiva de la competitividad. Antioquia: Universidad de Antioquia.

Castillo, J., \& García, M. (2013). Análisis de los factores explicativos de la rentabilidad de las empresas vinícolas de Castilla-La Mancha. Fc Uncuyo.

Castro, I. (2010). Variables internas para la optimizacion del sub sistema de mercadotecnia.

Chavarria, G. (2011). Estrategias promocional para incrementar las ventas.

Freire, C., Morán, S., Macas, J., \& Paucar, G. (2015). La falacia del liderazgo de genero. Revista científica de la facultad de ciencias económicas y administrativas, 17-28.

Fujii, D. (2004). LA TECNOLOGÍA Y EL ÉXITO INDUSTRIAL EN MÉXICO: UNA PROPUESTA DE DIVISIÓN SECTORIAL. Revista de Economía Mundial, 105-126.

Galarza, D. (2015). Estimación de la nota media del examen de matemáticas de 36 estudiantes mediante aplicación de medidas de dispersión estadísticas. Machala- Ecuador: Universidad Técnica de Machala.

García Ramos, J., Ruiz Garzón, G., \& Ramos, C. (2016). Estadística empresarial. Madrid-España: Universidad de Cadiz.

Guerrero, F. (2016). El análisis de regresión lineal y su incidencia en la estimación de los parámetros de la función consumo. Machala: Unidad Academica de Ciencias Empresariales.

Hong Zhang, G. (2016). Plan de negocios para la creación de una empresa ensambladora de pastillas de frenos en Ecuador. Quito: Universidad de las Américas.

Joekes, S., Righetti, A., \& Yacci, M. (2016). La importancia del error de medición en la variabilidad observada de los procesos. Revista de la Sociedad Argentina de Estadística, 10.

Joshi, M., Cahill, D., Sidhu, J., \& Kansal, M. (2013). El capital intelectual y financiero rendimiento : una evaluación del sector financiero australiano. 264-285.

Langebaek, A., \& Ortiz, J. (2007). Q de Tobin y Gobierno corporativo de las empresas listadas en 
bolsa. Borradores de Economía, 1-37.

Lind, D., Marchal, W. , \& Wathen, S. (2012). Estadistica aplicada a los negocios y la economia. Santa Fe.

Locia, E., Mederos, E., Morales, A., \& Rodríguez, J. (2014). Metodología para los procedimientos de solución de problemas sobre Ecuaciones Diferenciales. Revista Digital. Matemática, Educación e Internet., 2-6.

Menchaca, R. (2012). Intervalos de confianza:una aproximación intuitiva para el no-estadístico. Acta Médica Grupo Ángeles., 149.

Ricalde, D. (2013). Refórmese el art. 72 de la ley orgánica de defensa del consumidor, respecto a las sanciones a la publicidad engañosa por los proveedores, en garantía del consumidor en el Ecuador”. Loja - Ecuador: Universidad Nacional de Loja.

Rodriguez, J., \& Jimenez, E. (2016). Estadistica para Administracion. Mexico: Patria.

Rojo, J. (2007). Regresión lineal múltiple . Madrid: Instituto de Economía y Geografía .

Romero, R., \& Zunica, L. (2013). Metodo estadisticos para ingenieros. Valencia: Editorial Universitat Politècnica de València.

Ross, S., Westerfield, R., \& Jaffe, J. (2012). Finanzas corporativas. México D.F.: McGRAW-HILL.

Sáez, A. (2012). Apuntes de Estadística para Ingenieros. California: Universidad de Jaén.
Samaniego, A., \& Buenahora, M. (2016). Variables relacionadas con ansiedad social en adolescentes. Interacciones. Revista de Avances en Psicología, 114 .

Uriel , E. (2013). Regresión lineal múltiple: estimación y propiedades. Valencia: Universidad De Valencia.

Vasquez, R. (2016). Análisis Del Principio De Causalidad De Los Gastos De Publicidad De Las Empresas Del Sector Farmacéutico Peruano En La Determinación Del Impuesto A La Renta. Lima: Pontificia Universidad Catolica del Peru.

Velez, M. (2014). Prueba indiciaria en el delito de lavado de activos. Quito-Cuenca: Universidad de Azuay.

Zeitum, R., \& Salman, A. (2015). Dynamic performance evidence from GCC countries. EuroMed Journal of Business, 147-162.

Zurita, M. (2015). Análisis de las empresas metalmecánica Don Bosco y Mecanoplast Don Bosco y su respectiva propuesta de un plan de publicidad para mejorar su posicionamiento de mercado. Quito: Universidad Politecnica Salesiana sede Quito. 